\title{
Stability and predictability of orthognathic surgery
}

\author{
L'Tanya J. Bailey, DDS, MS ${ }^{a}$, Lucia H. S. Cevidanes, DDS, MS, PhD ${ }^{b}$, and William R. Proffit, \\ DDS, PhD $^{\mathrm{C}}$ \\ Department of Orthodontics, School of Dentistry, University of North Carolina, Chapel Hill
}

In assessing post-treatment stability in a group of treated patients, most of the change usually occurs in just a few of them. For this reason, it is highly misleading to use statistics based on normal distribution to describe posttreatment changes. With a normal distribution, the mean is the most likely indicator of what a patient would experience, and the clinician tends to think of it in just that way. But if essentially no change occurred in three fourths of the patients who underwent a certain type of treatment, and relatively large changes occurred in the one fourth who experienced change, the mean is highly misleading as an expectation of treatment response.

It is even more misleading to describe stability in terms of the percentage of treatment change that was retained at some follow-up time, as was done in many early articles on stability after orthognathic surgery. Reporting such percentages implies that the amount of relapse is directly related to the amount of treatment change: the more you changed it, the more it would relapse. In dentofacial patients, that almost never is the case. You simply cannot say that $80 \%$ of the amount of typical mandibular advancement will be retained, for instance, because, up to $8-10 \mathrm{~mm}$, posttreatment change (in the few patients who experience it) is relatively independent of the amount of advancement.

So how should stability data be reported? The best way is in terms of the percentage of the patients who have changes of a given magnitude. From that perspective, responses can be grouped as:

- Highly stable_-less than a $10 \%$ chance of significant posttreatment change

- Stable-less than a $20 \%$ chance of significant post-treatment change and almost no chance of major posttreatment change

- Stable if modified in a specific way (eg, rigid internal fixation [RIF] after surgery)

- Problematic: a considerable probability of major posttreatment change

In the real world, nothing is $100 \%$ successful, and high-risk procedures sometimes are quite successful. The clinician needs to know the odds of long-term stability and predictability with the possible treatment approaches, so that this information can guide the choice of treatment. The goal of this article is to put what we know in that context.

Copyright (c) 2004 by the American Association of Orthodontists.

Reprint requests to: L'Tanya J. Bailey, Department of Orthodontics, School of Dentistry, Chapel Hill, NC 27599-7450;

ltanya_bailey@dentistry.unc.edu.

${ }^{a}$ Associate professor and graduate program director.

b Postdoctoral fellow.

${ }^{\mathrm{c}}$ Kenan professor.

Presented at the American Association of Orthodontists/American Association of Oral and Maxillofacial Surgeons Symposium,

February 6-8, 2004; Palm Springs, Calif. 


\section{The hierarchy of stability}

The data for this article are taken from the University of North Carolina (UNC) Dentofacial Program database, which now contains over 3000 patients with initial records and over 1400 with at least 1 year follow-up. The database includes only patients with developmental deformities (no craniofacial anomalies or syndromes) treated with maxillary or mandibular orthognathic procedures.

Current data make it clear that, although modern orthognathic surgery can move the jaws and dentoalveolar segments, within limits, in any desired direction, there are major differences in stability and predictability. Based on this, it is possible to construct a hierarchy of procedures (Fig 1) and to group procedures into 4 major categories. ${ }^{1}$

Superior repositioning of the maxilla is the most stable orthognathic procedure, closely followed by mandibular advancement in patients with short or normal face height and less than $10 \mathrm{~mm}$ advancement. The UNC data for stability after mandibular advancement include only patients who met those criteria. Both these procedures can be highly stable, defined here as more than a $90 \%$ chance of less than $2 \mathrm{~mm}$ change at landmarks and almost no chance of more than $4 \mathrm{~mm}$ change during the first postsurgical year. Surgical repositioning of the chin via lower border osteotomy, the most prevalent adjunctive procedure, also is highly stable and predictable.

Advancement of the maxilla falls into the second category and can be described as stable. With forward movement of moderate distances $(<8 \mathrm{~mm})$, there is an $80 \%$ chance of less than $2 \mathrm{~mm}$ change, a $20 \%$ chance of 2-4 mm relapse, and almost no chance of more than $4 \mathrm{~mm}$ change. There is an important caveat, however. Downward movement of the maxilla is in the problematic category; if the maxilla is moved both forward and down, the vertical component is likely to relapse, although the horizontal component has a good chance of being retained. Correcting maxillary asymmetry usually involves moving 1 side up (and perhaps the other side down) to correct a canted occlusal plane and usually is done in conjunction with mandibular surgery. The maxillary component of asymmetry surgery also can be judged to be stable by the same criteria.

For acceptable stability, RIF does not appear to be required for procedures in the highly stable or stable categories. RIF does make a difference, however, when both jaws are repositioned simultaneously. The combination of maxilla up plus mandible forward and its Class III counterpart, maxilla forward plus mandible back, can be considered stable (by the same criteria listed above) only if RIF is used. Although the data are more limited, it appears that correction of major jaw asymmetry also falls into this category and that mandibular rigid fixation is important for asymmetry patients.

Three procedures are in the problematic category, defined as a $40 \%-50 \%$ chance of $2-4 \mathrm{~mm}$ postsurgical change and a significant chance of more than $4 \mathrm{~mm}$ change: mandibular setback, downward movement of the maxilla, and maxillary expansion. Even with these procedures, at least half of the patients experience essentially no postsurgical change. Every patient does not experience relapse, but there is a high chance of relapse. The goal, obviously, would be to take the additional steps to bring stability to more predictable levels when these surgical movements are needed.

\section{Long-term stability and condylar changes}

Surprisingly large changes in jaw dimensions and relationships, larger than those in untreated adults, occur beyond 1 year postsurgery for a few patients who have orthognathic surgery to correct Class II problems. ${ }^{2}$ Condylar resorption after mandibular advancement 
and relapse into anterior open bite have been reported as potential long-term clinical problems. ${ }^{3}$ Our long-term data now have placed these concerns in perspective: condylar changes occur in $5 \%-10 \%$ of patients who have surgery to advance the mandible, but a long-term increase in mandibular length (ie, growth at the condyles) is as likely as a decrease because of resorption, and, after open bite correction, a long-term increase in overbite is more likely than return of open bite. ${ }^{4}$ As yet, there are insufficient numbers of these patients with long-term follow-ups to allow subgroup analysis and the possible prediction of which patients might change in what way.

At this point, enough long-term data do not exist for confident prediction of the outcomes for treating some important dentofacial problems, in part because treatment has changed over time but also because these patients are a smaller proportion of the dentofacial population. This is particularly true for 2-jaw Class III surgery and asymmetry correction. It is important to extend this database, to reach the same level of certainty for these outcomes as for Class II surgery.

Transverse rotation of the condyles always accompanies ramus surgery to advance or set back the mandible, and transverse displacement also is highly likely. ${ }^{5}$ Studies using various methods have confirmed that condylar remodeling occurs after orthognathic surgery. ${ }^{6}$ Condylar remodeling has been of particular interest in patients with postsurgical temporomandibular joint problems.

Temporomandibular dysfunction does occur in a minority of orthognathic surgery patients and is thought to be related to how much the condyles have been displaced and particularly whether transverse displacement has occurred. ${ }^{7}$ Because placement of RIF devices can displace the condyles, RIF has been suggested as a factor in postsurgical temporomandibular dysfunction. ${ }^{8}$ Remodeling of the temporomandibular joint might or might not be associated with temporomandibular dysfunction after treatment.

Several investigators have used computerized axial tomography scans to evaluate changes in condylar position after various types of surgery, ${ }^{9,10}$ but there are no reports of how condylar changes seen in this way relate to morphologic or perceived outcomes. Thus, the extent to which remodeling at the condyles contributes to outcomes remains largely unknown. The recent development of cone-beam computed tomography equipment specialized for maxillofacial imaging ${ }^{11}$ is a relatively inexpensive, low-dose, and convenient way to follow condylar changes and their impact on jaw positioning and morphology in 3 dimensions. Currently, computed tomography scans are being collected for the UNC dentofacial patients to evaluate long-term condylar changes after orthognathic surgery.

Preliminary data from the computed tomography images suggest that much of the condylar rotation resulting in remodeling occurs from the surgical procedures, alone. Before-andafter-surgery superimpositions indicate that condylar changes can be quantified as to the amount of change and the timing of their occurrence (Figs 2-4). Further studies are required to determine the long-term effects of condylar changes relative to patient outcomes before definitive conclusions about condylar resorption and its relationship to the types of surgical procedures performed, and the patients with predisposing characteristics, can be assessed.

\section{Acknowledgments}

Supported in part by NIH grant DE-05215 from the National Institute of Dental Research.

We thank Debora Price for assistance with the database, Dr Ceib Phillips for statistical consultation, and the UNC oral and maxillofacial surgeons for their contributions through the years. 


\section{References}

1. Proffit WR, Turvey TA, Phillips C. Orthognathic surgery: a hierarchy of stability. Int J Adult Orthod Orthognath Surg. 1996; 11:191-204.

2. Schubert P, Bailey LJ, White RP, Proffit WR. Long-term cephalometric changes in untreated adults compared to those treated with orthognathic surgery. Int J Adult Orthod Orthognath Surg. 1999; 14:91-9.

3. De Clerq CA, Neyt LF, Mommaerts MY, Abeloos JV, De Mot BM. Condylar resorption in orthognathic surgery: a retrospective study. Int J Adult Orthod Orthog Surg. 1994; 9:233-40.

4. Proffit WR, Bailey LJ, Phillips C, Turvey TA. Long-term stability of surgical open bite correction by LeFort I oseotomy. Angle Orthod. 2000; 70:112-7. [PubMed: 10832998]

5. Becktor JP, Rebellato J, Becktor KB, Isaksson S, Vickers PD, Keller EE. Transverse displacement of the proximal segment after bilateral sagittal osteotomy. J Oral Maxillofac Surg. 2002; 60:395403. [PubMed: 11928096]

6. Tuinzing DB. Discussion of Harris et al. J Oral Maxillofac Surg. 1999; 57:654-5.

7. White CS, Dolwick MF. Prevalence and variance of temporomandibular dysfunction in orthognathic surgery patients. Int J Adult Orthod Orthognath Surg. 1992; 7:7-14.

8. Bouwman JP, Kerstens HC, Tuinzing DB. Condylar resorption in orthognathic surgery: the role of intermaxillary fixation. Oral Surg Oral Med Oral Pathol. 1994; 78:138-41. [PubMed: 7936579]

9. Kawamata A, Fujishita M, Nagahara K, Kanemata N, Niwa K, Langlais RP. Three-dimensional computed tomography evaluation of postsurgical condylar displacement after mandibular osteotomy. Oral Surg Oral Med Oral Pathol Oral Radiol Endod. 1998; 85:371-6. [PubMed: 9574943]

10. Harris MD, Van Sickels JE, Alder M. Factors influencing condylar position after the bilateral sagittal split osteotomy fixed with bicortical screws. J Oral Maxillofac Surg. 1999; 57:650-4. [PubMed: 10368087]

11. Mozzo MD, Procacci C, Tacconi A, Martini PT, Andreis IA. A new volumetric CT machine for dental imaging based on the cone-beam technique: preliminary results. Eur Radiol. 1998; 8:155864. [PubMed: 9866761] 


\title{
Surgical-Orthodontic Treatment:
}

\section{A Hierarchy of Stability}

\author{
Maxilla up \\ MORE \\ Mandible forward \\ Mexille forward \\ STABLEEI \\ PRSEDICTABLE

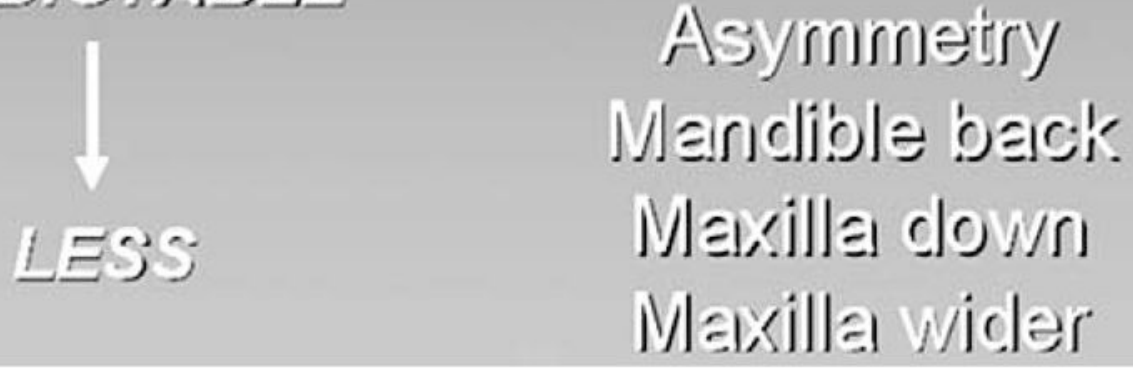 \\ MIX up + Mn forward \\ MX forwerd + Mln beck \\ Asymmetry \\ MJericlible besck. \\ Mexxille clow/s \\ Mexille wider
}

Fig 1.

Hierarchy of predictability and stability for orthognathic surgical procedures. 

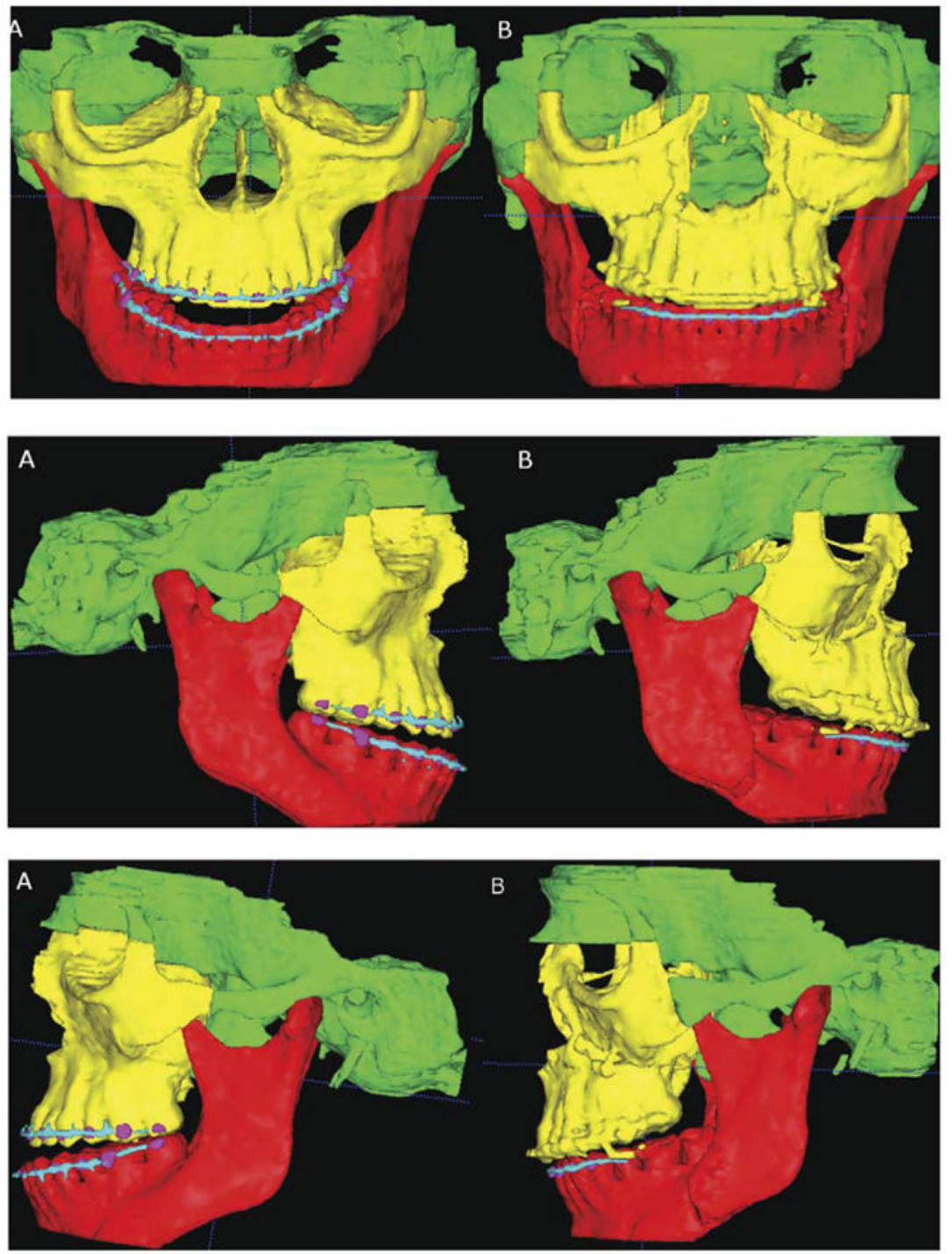

Fig 2.

Three-dimensional models of patient with asymmetric skeletal Class III relationship and open bite. A, 1 week before maxillary advancement with 2-piece LeFort I osteotomy and asymmetric expansion of right posterior segment, and mandibular setback with bilateral sagittal split osteotomy. B, 1 week after surgery. 


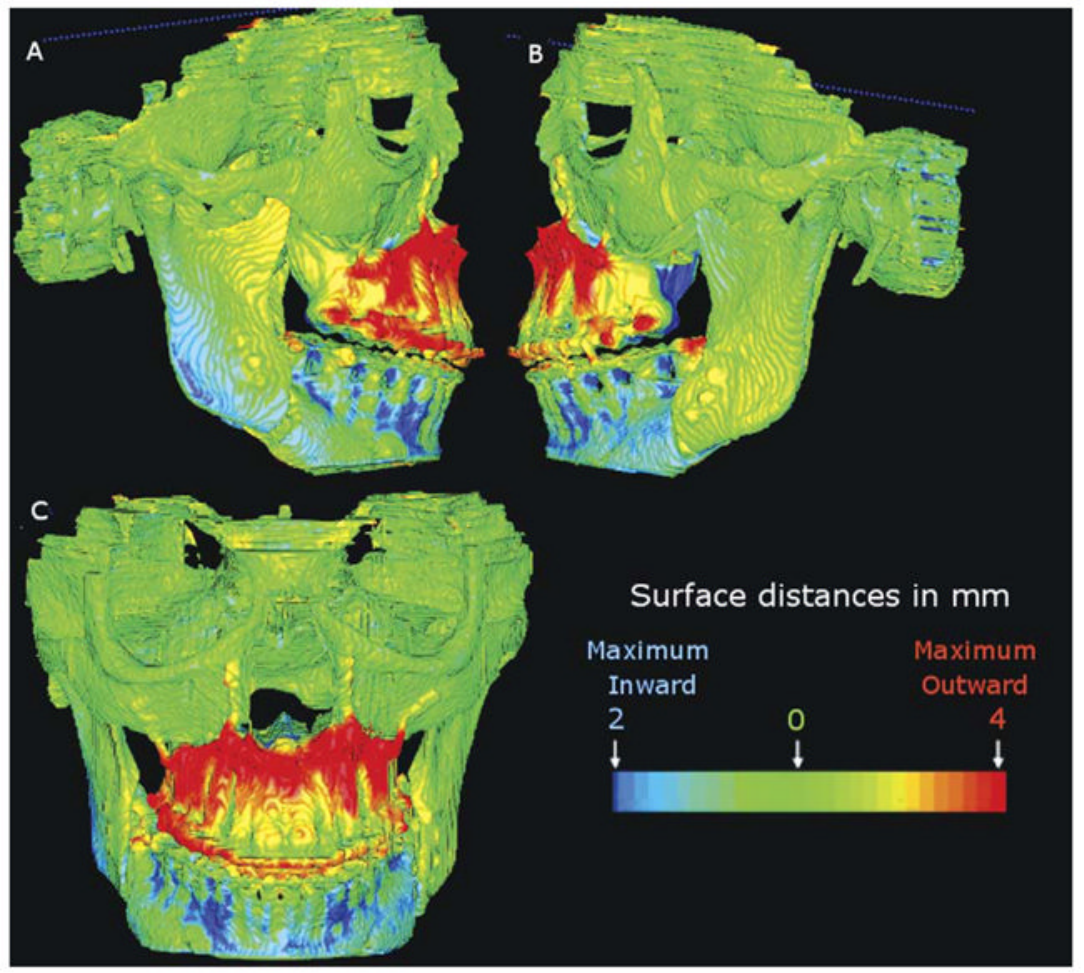

Fig 3.

Superimposition of presurgical and postsurgical 3-dimensional models registered on surface of cranial base. Cranial base color map is green $(0 \mathrm{~mm}$ surface distance) and shows adequate match of models for cranial base structures. Maxilla was brought forward (red) $4 \mathrm{~mm}$ along osteotomy, and mandibular corpus was set back (blue) $2 \mathrm{~mm}$. 

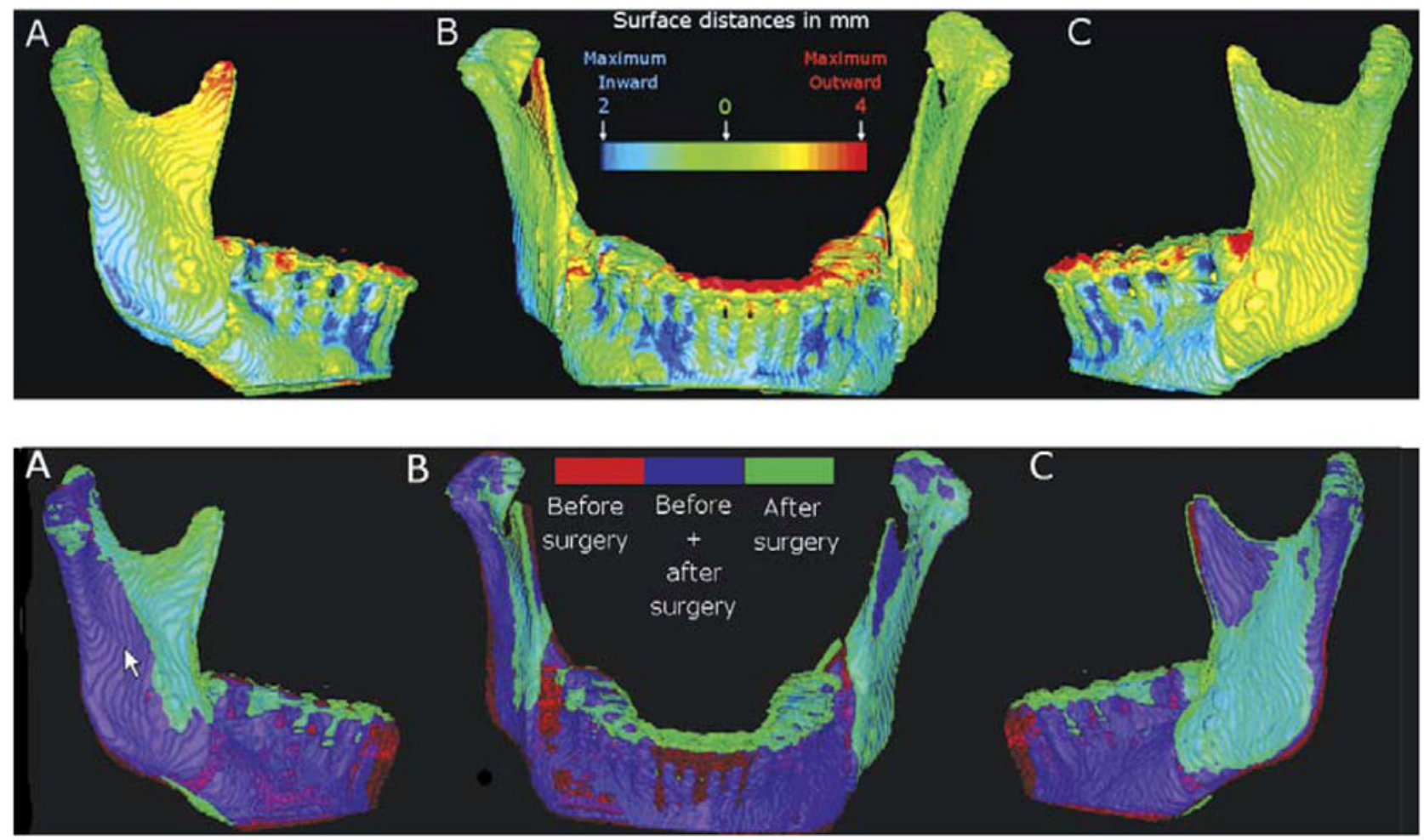

Fig 4.

Top, surface distances between presurgical and postsurgical mandibular models. Note that by using superimposition in Fig 3 and masking other anatomic structures, we can better visualize changes in mandibular corpus, ramus, and condyle. A, Minor (1 mm) forward/ mesial rotation of ramus on right side helped correct asymmetry; condyle was kept in same position. B, Frontal view shows mandibular setback ( $2 \mathrm{~mm}$, blue) and upward rotation (4 $\mathrm{mm}$, red). Anterior portion of lower border of mandible is not shown, so upward rotation cannot be assessed in this area. C, Color map for mandibular corpus is blue ( $2 \mathrm{~mm}$ setback); for ramus and condyle, it is close to green ( $0 \mathrm{~mm}$ surface distance), indicating that surgical movement on left side was restricted to mandibular corpus, and ramus and condyle were kept in position. Bottom, overlay of mandibular models. Red shows presurgical surgery models, green is postsurgical, blue indicates areas of overlap. 